\title{
The Effectiveness of Mobile-Learning in Teaching Corrective Exercises to Scoliosis Patients: A Quasi- Experimental Study
}

Taha Samad-Soltani ( $\sim$ Samadsoltani@tbzmed.ac.ir)

Tabriz University of Medical Sciences https://orcid.org/0000-0002-1555-4714

Arefeh heshmati

Tabriz University of Medical Sciences

Hakimeh Hazrati

Tabriz University of Medical Sciences

Zahra Salahzade Salahzade

Tabriz University of Medical Sciences

Peyman Rezaei hachesu

Tabriz University of Medical Sciences

Nushin khalili ekrami

Tabriz University of Medical Sciences

\section{Research}

Keywords: Mobile-Learning, Corrective Exercise, Scoliosis, App, Quasi-Experimental Study

Posted Date: November 15th, 2021

DOI: https://doi.org/10.21203/rs.3.rs-931752/v2

License: (c) (i) This work is licensed under a Creative Commons Attribution 4.0 International License.

Read Full License 


\section{Abstract}

Background: Mobile-learning (M-learning) has recently provided new opportunities and facilitated patient education due to its attractiveness and ease of access. As a result, the present study aimed to design an application (app) and evaluate the effectiveness of M-learning in teaching CEx to patients suffering from scoliosis.

Methods: This quasi-experimental study was fulfilled in two phases, namely, app design and educational intervention among scoliosis patients, and then reflections on the effectiveness of the app and educational intervention implementation concerned. Within the implementation step, the scoliosis patients referred to the rehabilitation center affiliated with Tabriz University of Medical Sciences, Tabriz, Iran, were divided into case and control groups upon imaging and grading their spine curvature. The effectiveness of the educational intervention was also assessed through the rate of recovery in spine curvature and users' opinions and satisfaction with the app usability via the User Experience Questionnaire (UEQ).

Results: In this sense, the accuracy index was equal to $100 \%$ based on physiotherapists' approval. The level of satisfaction with the educational intervention in the case group with a mean value of $97 \%$ was also higher than that in the controls (88\%). Moreover, the values of the Cob angle change in the case group were significantly higher than that in the controls. Based on the analysis of the UEQ outcomes, the highest and the lowest levels of satisfaction among the users were associated with the "perspicuity" and "stimulation" dimensions of the app, respectively.

Conclusion: Given that the new models of care are today mostly focused on the role of patients, moving toward patient self-management, the app designed here could be utilized as a model for developing broader similar systems and programs for treatment management, patient monitoring, emergency response, personalized health monitoring, and widespread access to health care information.

\section{Key Message}

- The widespread use of smartphones and their increasing availability and appeal in markets across the world have created many new opportunities to provide health services via mobile phones

- In a small sample this study shows that patient's participation and recovery increased after use the application

- This study shows the mean positive effect of the application on patients after the intervention and use of the application is more than before the intervention and not using the program.

\section{Background}

Scoliosis is known as one of the most common Musculoskeletal Disorders affecting the spine. If not diagnosed early enough, this disorder can bring about problems in patients' appearance and sitting 
positions, and even lead to lung diseases and heart problems in more severe cases $(1,2)$. Physiotherapists and orthopedic specialists accordingly are making efforts to exploit appropriate methods to treat this disorder. Meanwhile, non-surgical procedures are much more emphasized, particularly corrective exercises (CEx, viz. corrective movements)(3).Performing such movements during home exercises is easier said than done (4). and the proper execution of such exercises is of special importance (5). As these movements must be done repeatedly, they may make patients exhausted and thus compel them to avoid performing CEx correctly, with reduced therapeutic effects. Therefore, it is critical to stimulate patients with spine curvature, especially scoliosis, and monitor how they do such exercises (6). Nowadays, mobile health (mHealth) technologies help rehabilitation professionals through decision support systems in health care and provide tools for monitoring the effects of home exercise programs, collecting reliable results or vital signs, giving feedback on the body postures and mechanics, and delivering educational materials and the messages of encouragement to patients. Therefore, they can increase safety awareness, diminish costs, and even improve treatment quality by enhancing patient adherence to health care strategies (7-11)Considering its attractiveness, ease of access, and ability to install decision support apps in health care, mobile technology as one of the mHealth sub-branches, also makes it possible to focus on patient-centeredness, with the lowest costs and no need for direct supervision by health personnel, which can ultimately facilitate patient education. Thus, the present study aimed to design an application (app) and evaluate the effectiveness of M-learning in teaching CEx to scoliosis patients using a quasi-experimental research design. Benefiting from the capabilities of decision support systems and clinical solutions, therapists' decisions regarding the correct diagnosis of the type of scoliosis and the right exercise for the type of scoliosis could be accordingly reinforced by producing knowledge-based content and incorporating it into a mobile app.

\section{Materials And Methods}

This study was to create a comprehensive home exercise app for scoliosis patients with the capability to run on smartphones with the Android operating system. This quasi-experimental study was fulfilled in two phases, namely, app design and educational intervention in patients suffering from scoliosis, and then reflections on the effectiveness of the app and educational intervention implementation concerned.

During the app design and implementation, the features of the app and its content were determined through a review of the related literature along with a focus group discussion. To indicate the information needs and the capabilities of the app, three focus group sessions were further conducted with the presence of six specialists in the field of health informatics, health information management, physiotherapy, exercise therapy, and the Android programming. In order to establish the app content (namely, exercises appropriate to the type of scoliosis); the Schroth method was utilized under the supervision of rehabilitation specialists in addition to the literature review. The exercises were accordingly set in five areas, i.e., hanging, mobilization, shaping, stretch, and education. The proper exercises in each area are illustrated in Table 1. 
Table 1

Areas and types of CEx for scoliosis patients

\begin{tabular}{|c|c|c|c|}
\hline & & & Exercise \\
\hline \multirow[t]{2}{*}{1} & \multirow[t]{2}{*}{ Hanging } & $\begin{array}{l}\text { 3- } \\
\text { curve }\end{array}$ & Butterfly, Air cycling, Pendulum legs, Abdominal muscle strengthening \\
\hline & & $\begin{array}{l}4- \\
\text { curve }\end{array}$ & Butterfly, Air cycling, Abdominal muscle strengthening \\
\hline \multirow[t]{2}{*}{2} & \multirow[t]{2}{*}{ Mobilization } & $\begin{array}{l}\text { 3- } \\
\text { curve }\end{array}$ & $\begin{array}{l}\text { Squat with wall-bar, Sagittal circling with the trunk, Diagonal circling } \\
\text { with the trunk, Sagittal circling, Chest-straddle position on the chair, } \\
\text { Raising hands while sitting on the floor }\end{array}$ \\
\hline & & $\begin{array}{l}\text { 4- } \\
\text { curve }\end{array}$ & $\begin{array}{l}\text { Squat with wall-bar, Raising hands while sitting on the floor, Chest- } \\
\text { straddle position on the chair, Sagittal circling with the trunk, Sagittal } \\
\text { circling }\end{array}$ \\
\hline \multirow[t]{3}{*}{3} & \multirow[t]{3}{*}{ Shaping } & $\begin{array}{l}\text { 3- } \\
\text { curve }\end{array}$ & $\begin{array}{l}\text { Great arch, Oblique traction, Neck posture CEx, Grabbing the back of the } \\
\text { seat from behind, Head under the desk, Rotational sitting with a Swiss } \\
\text { ball, Rotational sitting, Side bridge (3-curve scoliosis), Putting the weight } \\
\text { on one foot, Corrective rotation in the pelvis, Muscle cylinder exercise }\end{array}$ \\
\hline & & & $\begin{array}{l}\text { Shaping with wall-bar, Raising the right sulcus shaping (3-curve } \\
\text { scoliosis), Lowering the right sulcus in the standing position, Hip shift } \\
\text { correction }\end{array}$ \\
\hline & & $\begin{array}{l}\text { 4- } \\
\text { curve }\end{array}$ & $\begin{array}{l}\text { Great arch, Rotational sitting with a Swiss ball, Head under the desk, } \\
\text { Grabbing the back of the seat from behind, Neck posture CEx, Oblique } \\
\text { traction, Correction of lumbosacral curvature (4-curve scoliosis), Side } \\
\text { bridge (4-curve scoliosis), Correction of lumbosacral curvature } 2 \text { (4-curve } \\
\text { scoliosis), Raising the right sulcus, Shaping with wall-bar, Raising the } \\
\text { right sulcus, Lowering the right sulcus, 3D made easy (4-curve scoliosis) }\end{array}$ \\
\hline \multirow[t]{2}{*}{4} & \multirow[t]{2}{*}{ Stretch } & $\begin{array}{l}\text { 3- } \\
\text { curve }\end{array}$ & $\begin{array}{l}\text { Pulling toward wall-bar, Resistive pelvis shift, Strap-holding exercise, } \\
\text { Lifting the trunk with two bars, Stretching in the sitting position using a } \\
\text { bar, Prostration in the kneeling position, Exercise to stretch and } \\
\text { strengthen the back in the low sliding position }\end{array}$ \\
\hline & & $\begin{array}{l}\text { 4- } \\
\text { curve }\end{array}$ & $\begin{array}{l}\text { Pulling toward wall-bar, Strap-holding exercise, Lifting the trunk with two } \\
\text { bars, Stretching in the sitting position using a bar, Prostration in the } \\
\text { kneeling position, Exercise to stretch and strengthen the back in the low } \\
\text { sliding position }\end{array}$ \\
\hline \multirow[t]{2}{*}{5} & \multirow[t]{2}{*}{ Education } & $\begin{array}{l}\text { 3- } \\
\text { curve }\end{array}$ & $\begin{array}{l}\text { Sitting training (3-curve scoliosis), Sitting training (3-curve scoliosis), } \\
\text { Standing training with flat back (3-curve scoliosis), Standing training } \\
\text { with kyphosis (3-curve scoliosis) }\end{array}$ \\
\hline & & $\begin{array}{l}\text { 4- } \\
\text { curve }\end{array}$ & Sitting training (4-curve scoliosis) \\
\hline
\end{tabular}

At the next step, a person who had already mastered the given exercises did them for 20 hours. The CEx was then filmed using a high-quality Sony FDR-AX33 camera. Upon recording, the videos were edited via the Adobe After Effects CC 2017 v14.1 software. In addition to the videos, audios were also applied for further learning purposes. Rules, texts, and exercises were subsequently approved by some experts in this 
field, and then the design process was completed using the Java programming language and the Extensible Markup Language (XML) to design the user interface.

With reference to the agreement by the members of the panel of experts, the personal information in the designed app included gender and a unique patient code, the clinical information was about defining the type of scoliosis (viz. three and four curvatures), and the app capabilities contained CEx time reminder, proper CEx selection for spine curvature for the therapist, as well as texts and images.

During the app design phase, the functional requirements of the use case and activity diagrams were correspondingly plotted in the Visual Paradigm software. The relationships between the actors and use cases are displayed in Fig. 1. Two activity diagrams were also designed to explain the system processes in Fig. 2 and Fig. 3.

The user interface designed in this study comprised of login, registration, primary data entry, homepage, viewing exercises, viewing entered data, intervention, evaluation, displaying the recommended list of exercises, and other items. After designing the app, the software defects were also fixed and upgraded during the testing process once they were approved by the experts in this field.

As the app was installed on the therapist's smartphone, he/she could register in it. The information entered by the therapist, included name, medical education number, role, as well as username and password. After registration, he/she could register patients in the app by clicking on the New Patient (+) option. Then, by clicking on the Register option, the patient's unique code could be received. By selecting the patient, the type of scoliosis was then selected (namely, three or four curves), then the recommended list of the exercises could be provided by the app. The therapist could accordingly select and set the desired exercises. After installing the app, the patient could also register on their smartphone. First, they could enter the unique code by clicking on the Sign Up option and clicking the Submit option. In the subsequent step, they could register and browse the exercises in the app. It should be noted that the CEx could be downloaded once, and played several times (Fig. 4).

At the implementation step, the scoliosis patients referred to the rehabilitation center affiliated with Tabriz University of Medical Sciences, Tabriz, Iran, were divided into case and control groups after imaging and grading their spine curvature. The inclusion criteria for this purpose were women, aged 15-30, with the diagnosis of idiopathic scoliosis with the Cobb angle (20-40 degrees), no history of rehabilitation and spinal surgery, being literate, and having a smartphone with the Android operating system.

Specialists in physiotherapy, medical informatics, and health information management also formed the study population in the evaluation phase. The study sample accordingly consisted of two specialists in the field of physiotherapy, one person in the field of medical informatics, and one case in the field of health information management, showing willingness to participate in the study, randomly selected using the convenience sampling method. 
The Cobb method was also applied to grade the spine curvature (12). This was the most widely used measurement to analyze the lateral curvatures of the spine based on radiographic images. All the participants also received their CEx for twelve weeks. The only difference was how the exercises were presented to both study groups. In this respect, the participants in the case group received their exercises in a mobile-based app. Such exercises were approved and adjusted by the therapist. The home exercise program was further performed during the study period and at least five times a week. The participants in the control group only went to the physiotherapy clinic and performed CEx in the presence of a therapist. Then, they received paper handouts containing the pictures of the exercises and accordingly did the exercises at home.

After the intervention, the rates of recovery and learning in both study groups were measured. To determine the extent of the patients' recovery rate, their Cob angles were measured before and after CEx in the study groups. The data obtained from the Cobb angle were then analyzed using the Wilcoxon signed-rank test in the SPSS Statistics software. To measure the rate of learning, the exercises were also performed by both groups in the presence of an expert in this field and the data were qualitatively collected according to the relevant expert opinions with a range of good, moderate, and bad.

The users' opinions about the app usability and their level of satisfaction were further evaluated through the User Experience Questionnaire (UEQ) within six dimensions (i.e., attractiveness, perspicuity, efficiency, dependability, stimulation, and novelty). Mean and standard deviation (SD) among the descriptive statistics were accordingly used to report patient satisfaction. In this questionnaire, the mean score between -0.8 and +0.8 was further considered as a neutral evaluation, the value greater than 0.8 was deemed as a positive evaluation, and that smaller than -0.8 represented a negative evaluation (13).

\section{Results}

The study population included all patients referred to specialized physiotherapy and orthopedic clinics and the study sample included 20 women aged 15-30 with a diagnosis of idiopathic scoliosis referred to physiotherapy clinics affiliated with Tabriz University of Medical Sciences, Tabriz, Iran, during the second half of 2018.

The mean rate of the Cob angle change in the case group showed better recovery and was significantly higher than that in the control group. Considering $95 \%$ confidence interval $(\mathrm{Cl})$, the app had a positive effect on the patients' recovery, and a significant difference was further observed between the results before and after the educational intervention in both groups $(\mathrm{P}<0.05, \mathrm{Cl}=95 \%)$ (Table 2$)$. 
Table 2

Change in the Cobb angle before and after the educational intervention in study groups

\begin{tabular}{|llll|}
\hline Groups & $\begin{array}{l}\text { Before CEx } \\
\mathbf{N}(\text { mean } \pm \text { SD })\end{array}$ & $\begin{array}{l}\text { After CEx } \\
\mathbf{N}(\text { mean } \pm \text { SD })\end{array}$ & P-value \\
\hline Case & $10(30.10 \pm 5.68)$ & $10(17.60 \pm 10.56)$ & 0.004 \\
\hline Control & $10(29.80 \pm 10.56)$ & $10(22.30 \pm 10.13)$ & 0.008 \\
\hline
\end{tabular}

The rate of learning and the practice of CEx by the patients in both groups were also determined blindly by a rehabilitation specialist through observing the exercises performed by the patients ranged at good, moderate, and bad. As well, $80 \%$ of the patients in the case group received a good quality score for the exercises after using the app, but this value was equal to $30 \%$ in the control group.

Finally, 10 users of the app evaluated it in six dimensions (viz. attractiveness, perspicuity, efficiency, dependability, stimulation, and novelty) using the UEQ. The results of this evaluation demonstrated that the highest and the lowest levels of satisfaction among the users were associated with the "perspicuity" and "stimulation" dimensions of the app (Fig. 5).

\section{Discussion}

Musculoskeletal disorders can cause not only physical disabilities but also unpredictable mental and social problems. As many researchers have suggested, mobile applications can very well address the need of patients with musculoskeletal disorders to education. Health promotion applications designed to support disease prevention and promote healthy behaviors are among the most commonly downloaded applications(14).This study aimed to develop a comprehensive home exercise program by providing interactive platforms for disease management by scoliosis patients using smartphones with the Android operating system. The study results showed that the designed app improved this medical condition further by shrinking the Cobb angle in the case group compared with the controls. In Villani et al., remote rehabilitation had also positively affected the patients with musculoskeletal disorders (15). As well, Lambert et al. had found that the cases with musculoskeletal disorders using mobile-based exercise programs at home had significantly adhered to CEx than those who had used only paper handouts to exercise (16). The use of multimedia learning tools in patients with a stoma had similarly improved patient preparation in a positive manner through providing health information, teaching coping strategies, facilitating self-care behaviors, increasing awareness, and improving their self-care and behavioral attitudes (17). Also Christopher Tack explained that digital behavior change interventions can enhance self-management in individuals with musculoskeletal conditions.(18). More patient adherence to CEx was possibly due to some of the features of super-electronic health, such as increased accessibility, scalability and flexibility, use for multiple health care providers unlike other electronic health systems, and maintainability (13). Therefore, they can be employed as a potential solution in the health care industry (13). Baek et al. conducted a research to compare methods using booklets and apps to identify effective ways of providing self-exercise programs to female farmers with musculoskeletal health problems. They 
recruit 200 female farmers. They emphasized that the smartphone-based interventions may be a useful method for managing musculoskeletal health in farmers living in rural areas and can provide more satisfaction, better quality of life, and more coherent in exercise following (19). Also, a pilot feasibility study indicated that the rehabilitation apps are safe, pleasant, and feasible for older adults to participate in a home-based, self-care, and multicomponent exercise program targeting musculoskeletal disorders and function that was delivered and monitored remotely by exercise experts(20). As a whole, the results of the present study showed a relatively high level of user satisfaction with the app although the level of satisfaction was lower than other cases in the dimension of "stimulation", even if it could play a leading role in improving self-efficacy and better perceptions in patients (14).

There are various approaches, ranging from common videoconferencing to complex sensor and gamification technologies for telerehabilitation, that assist patients with musculoskeletal disorders when exercising at home. Most of them are not practical in the real world routines. The reasons are varying prototype status, different and expensive instruments, missing telerehabilitation aspects, additional cost, and few efficacy studies. Therefore, Prototype testing, usability, and acceptance tests under real-life conditions are required (21). Interactive tools, sensors, and business intelligence techniques can be thus used to improve patients' stimulation. In the study by Feistritzer-Gröbl et al., the application of computer games had accordingly increased patients' stimulation and augmented it (4). However, it does lead to some challenges. Biebl et al. reported that in previous studies, the researchers have used 3D sensors such as the Microsoft Kinect and other posture capture systems to assess pose during exercise and give feedback to patients if correction was required. However, these market-based products are expensive and need extensive external hardware and multimedia set, and thus have limited scalability and feasibility in providing pervasive access to digital rehabilitation, especially in developing countries (22). Also, Virtual Reality(VR) has major potential to provide high immersion of patients in the virtual exergames of rehabilitation. However, it leads to additional cost of head mounted display (VR headset) to each person. We performed similar studies and the cost-effectiveness is a very important factor in VR applicability (23). In the current study, we had many technical and technological options, but due to limited resources available to patients and minimize cost imposition to them; the design was limited to the smartphones capabilities.

\section{Conclusion}

Given that the new models of care are today mostly focused on the role of patients, moving toward patient self-management, the app designed here could be utilized as a model for developing broader similar systems and programs for treatment management, patient monitoring, emergency response, personalized health monitoring, and widespread access to health care information in the low-income countries.

\section{Declarations}

\section{Ethics approval and consent to participate}


This study was reviewed and approved by the Ethics Committee of Tabriz University of Medical Sciences (IR.TBZMED.REC.60315). All patients provided written informed consents. All methods were performed in accordance with the relevant guidelines and regulations.

\section{Consent for publication}

Not applicable

\section{Availability of data and materials}

The datasets used and/or analyzed during the current study are available from the corresponding author on reasonable request.

\section{Competing interests}

The authors declare that they have no competing interests.

\section{Funding}

This study was funded by Vice-Chancellor for Research and Technology of Tabriz University of Medical Sciences, Tabriz, Iran.

\section{Authors' contributions}

TS,PR,ZS and AH conceived and designed the study. TS,PR,ZS and AH analyzed and interpreted the data, and drafted the manuscript. TS, PR, ZS, HH, NE and AH were involved in the composition of the study tool, supervision of the research process and critical revision and review of the manuscript. All the authors read and approved the final manuscript.

\section{Acknowledgment}

Authors would like to thank the Tabriz University of Medical Sciences under Grant 60315

\section{References}

1. Anitha $H$, Prabhu G. Identification of apical vertebra for grading of idiopathic scoliosis using image processing. Journal of digital imaging. 2012;25(1):155-61.

2. Johari J, Sharifudin MA, Ab Rahman A, Omar AS, Abdullah AT, Nor S, et al. Relationship between pulmonary function and degree of spinal deformity, location of apical vertebrae and age among adolescent idiopathic scoliosis patients. Singapore medical journal. 2016;57(1):33.

3. Białek M. Conservative treatment of idiopathic scoliosis according to FITS concept: presentation of the method and preliminary, short term radiological and clinical results based on SOSORT and SRS criteria. Scoliosis. 2011;6(1):25. 
4. Feistritzer-Gröbl P, Nischelwitzer A, Saraph V, Holzer H, editors. Game Based Physiotherapy for Treatment of Children with Juvenile Idiopathic Scoliosis. Proceedings of Measuring Behavior; 2012: Citeseer.

5. Negrini S, Zaina F, Romano M, Negrini A, Parzini S. Specific exercises reduce brace prescription in adolescent idiopathic scoliosis: a prospective controlled cohort study with worst-case analysis. Journal of rehabilitation medicine. 2008;40(6):451-5.

6. Wibmer C, Groebl P, Nischelwitzer A, Salchinger B, Sperl M, Wegmann H, et al. Video-game-assisted physiotherapeutic scoliosis-specific exercises for idiopathic scoliosis: case series and introduction of a new tool to increase motivation and precision of exercise performance. Scoliosis and spinal disorders. 2016;11(1):44.

7. Peleg M. Computer-interpretable clinical guidelines: a methodological review. Journal of biomedical informatics. 2013;46(4):744-63.

8. Goud R, Hasman A, Peek N. Development of a guideline-based decision support system with explanation facilities for outpatient therapy. Computer methods and programs in biomedicine. 2008;91(2):145-53.

9. Samad-Soltani T, Ghanei M, Langarizadeh M. Development of a fuzzy decision support system to determine the severity of obstructive pulmonary in chemical injured victims. Acta Informatica Medica. 2015;23(3):138.

10. Wollersheim H, Burgers J, Grol R. Clinical guidelines to improve patient care. 2005.

11. Bates DW, Kuperman GJ, Wang S, Gandhi T, Kittler A, Volk L, et al. Ten commandments for effective clinical decision support: making the practice of evidence-based medicine a reality. Journal of the American Medical Informatics Association. 2003;10(6):523-30.

12. KURU ÇOLAK T, Yeldan I, Dereli E, Ozdincler A, Dikici F, Colak I. The efficacy of three-dimensional Schroth exercises in adolescent idiopathic scoliosis: A randomised controlled clinical trial. 2016.

13. Desteghe L, Kluts K, Vijgen J, Koopman P, Dilling-Boer D, Schurmans J, et al. The health buddies app as a novel tool to improve adherence and knowledge in atrial fibrillation patients: a pilot study. JMIR mHealth and uHealth. 2017;5(7):e98.

14. Fitzgerald $M$, McClelland $T$. What makes a mobile app successful in supporting health behaviour change? Health education journal. 2017;76(3):373-81.

15. Villani GQ, Villani A, Zanni A, Sticozzi C, Maceda DP, Rossi L, et al. Mobile health and implantable cardiac devices: Patients' expectations. European journal of preventive cardiology. 2019;26(9):9207.

16. Lambert TE, Harvey LA, Avdalis C, Chen LW, Jeyalingam S, Pratt CA, et al. An app with remote support achieves better adherence to home exercise programs than paper handouts in people with musculoskeletal conditions: a randomised trial. Journal of physiotherapy. 2017;63(3):161-7.

17. Lo SF, Wang YT, Wu LY, Hsu MY, Chang SC, Hayter M. Multimedia education programme for patients with a stoma: effectiveness evaluation. Journal of advanced nursing. 2011;67(1):68-76. 
18. Tack C. A model of integrated remote monitoring and behaviour change for osteoarthritis. BMC Musculoskeletal Disorders. 2021;22(1):1-8.

19. Baek S, Kim G, Park H-w. A mobile delivered self-exercise program for female farmers. Medicine. 2020;99(52).

20. Daly RM, Gianoudis J, Hall T, Mundell NL, Maddison R. Feasibility, Usability, and Enjoyment of a Home-Based Exercise Program Delivered via an Exercise App for Musculoskeletal Health in Community-Dwelling Older Adults: Short-term Prospective Pilot Study. JMIR mHealth and uHealth. 2021;9(1):e21094.

21. Elgert L, Steiner B, Saalfeld B, Marschollek M, Wolf K-H. Health-Enabling Technologies to Assist Patients With Musculoskeletal Shoulder Disorders When Exercising at Home: Scoping Review. JMIR Rehabilitation and Assistive Technologies. 2021;8(1):e21107.

22. Biebl JT, Rykala M, Strobel M, Bollinger PK, Ulm B, Kraft E, et al. App-Based Feedback for Rehabilitation Exercise Correction in Patients With Knee or Hip Osteoarthritis: Prospective Cohort Study. Journal of Medical Internet Research. 2021;23(7):e26658.

23. Cesco A, Ballardin F, Marfia G, editors. CeVRicale: A VR app for Cervical Rehabilitation. 2021 IEEE Conference on Virtual Reality and 3D User Interfaces Abstracts and Workshops (VRW); 2021: IEEE.

\section{Figures}

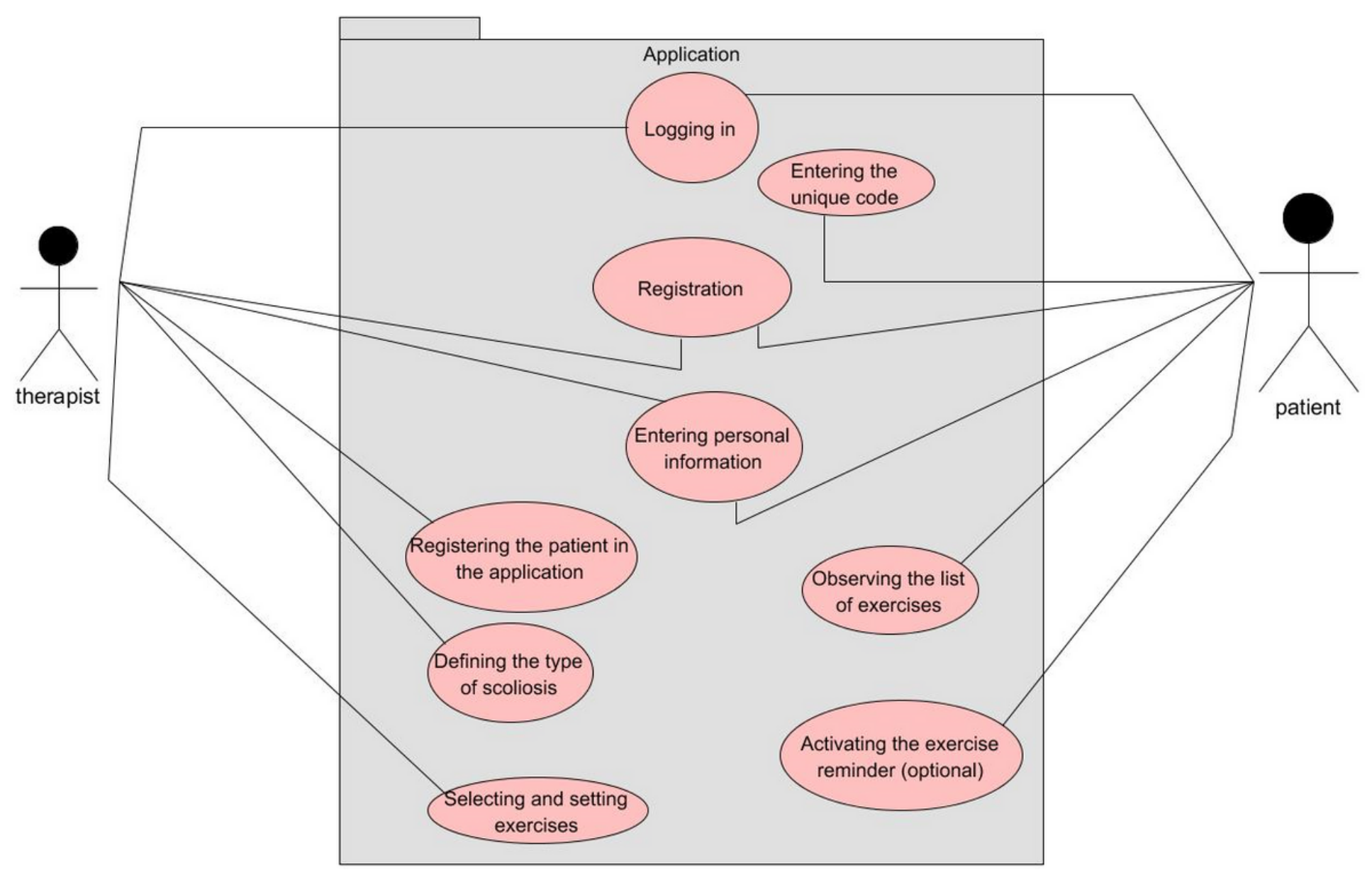


Figure 1

Use case diagram

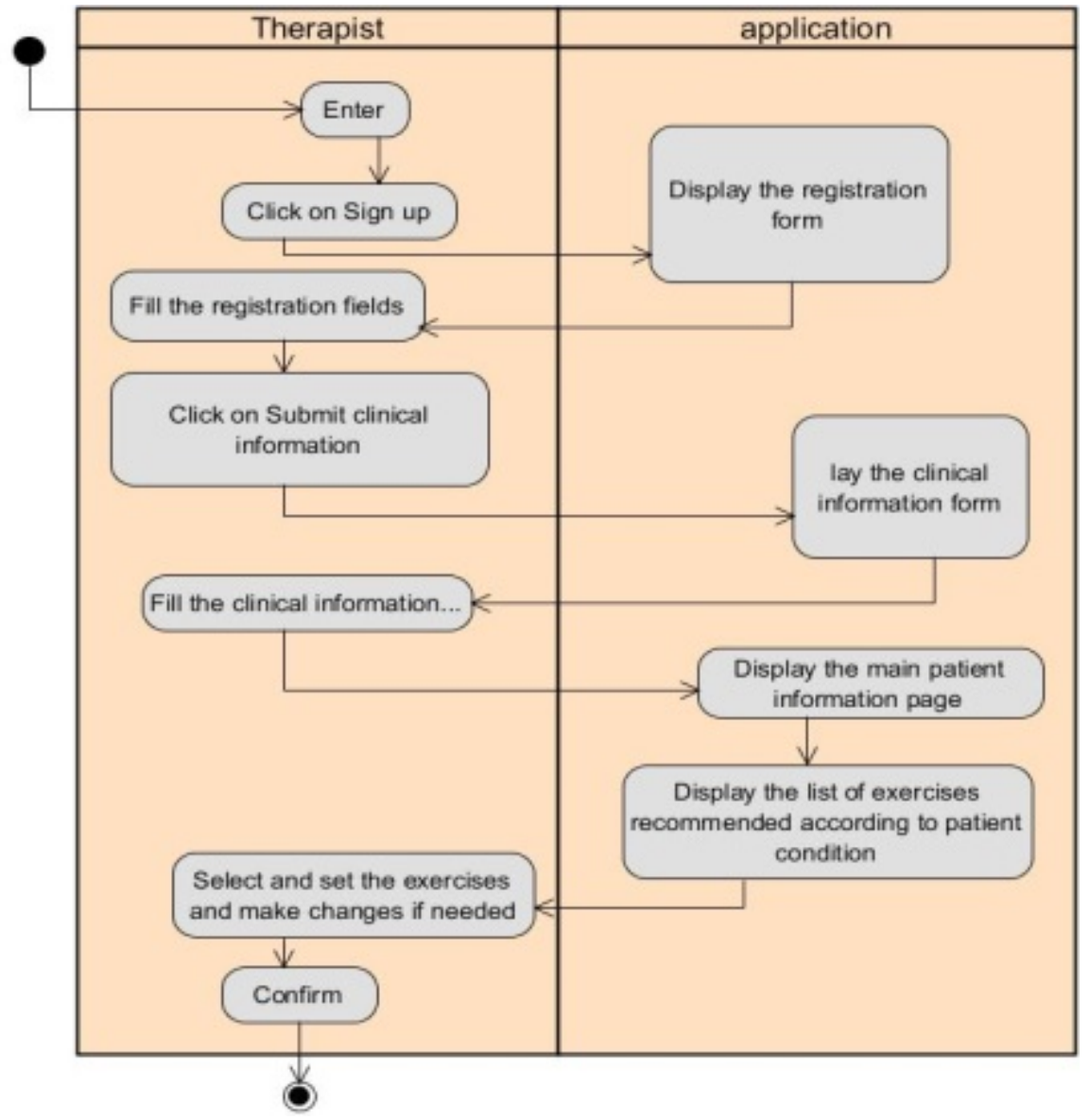

\section{Figure 2}

Activity diagram (interactions between a therapist and the app) 


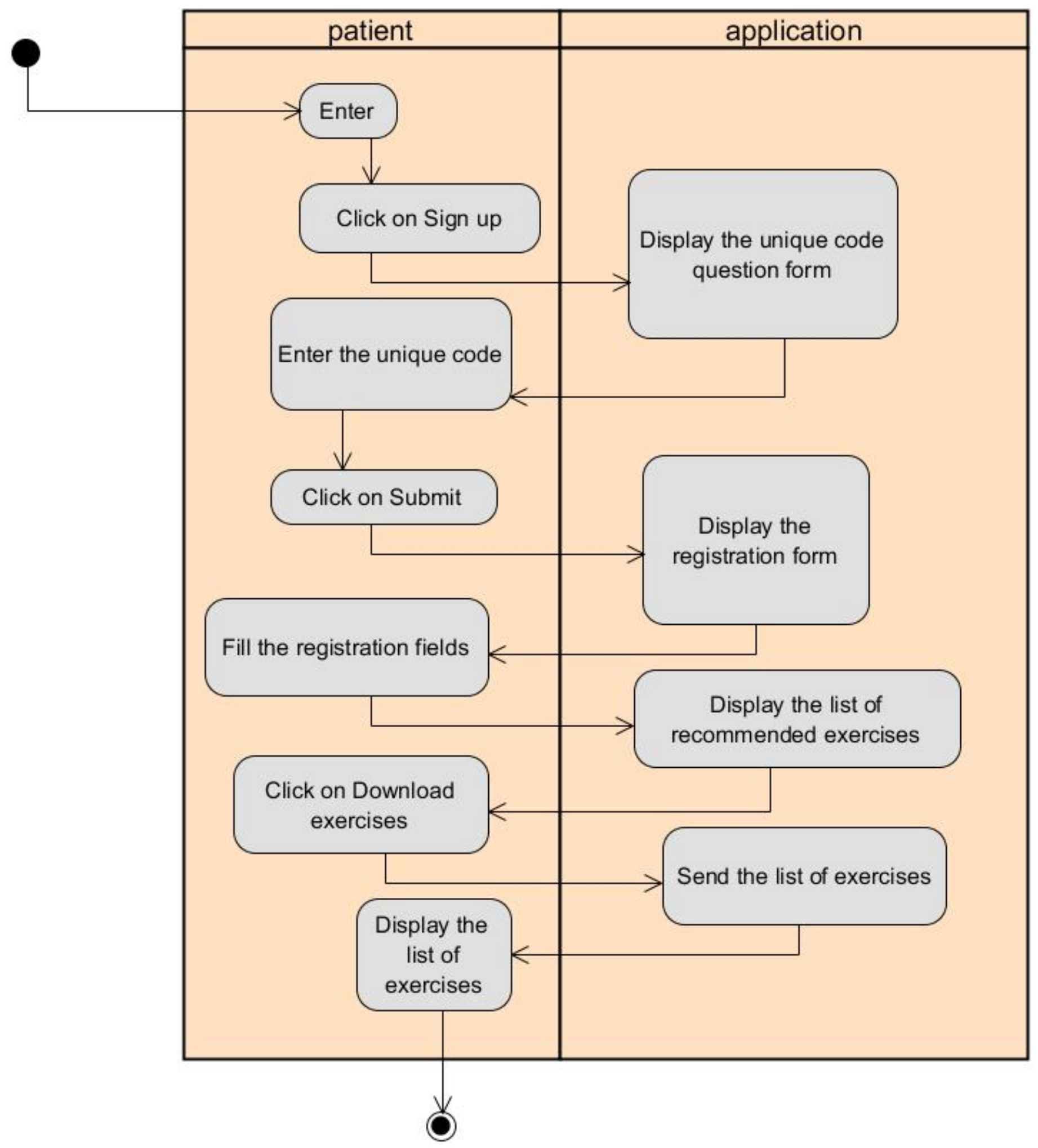

Figure 3

Activity diagram (interactions between a patient and the app) 


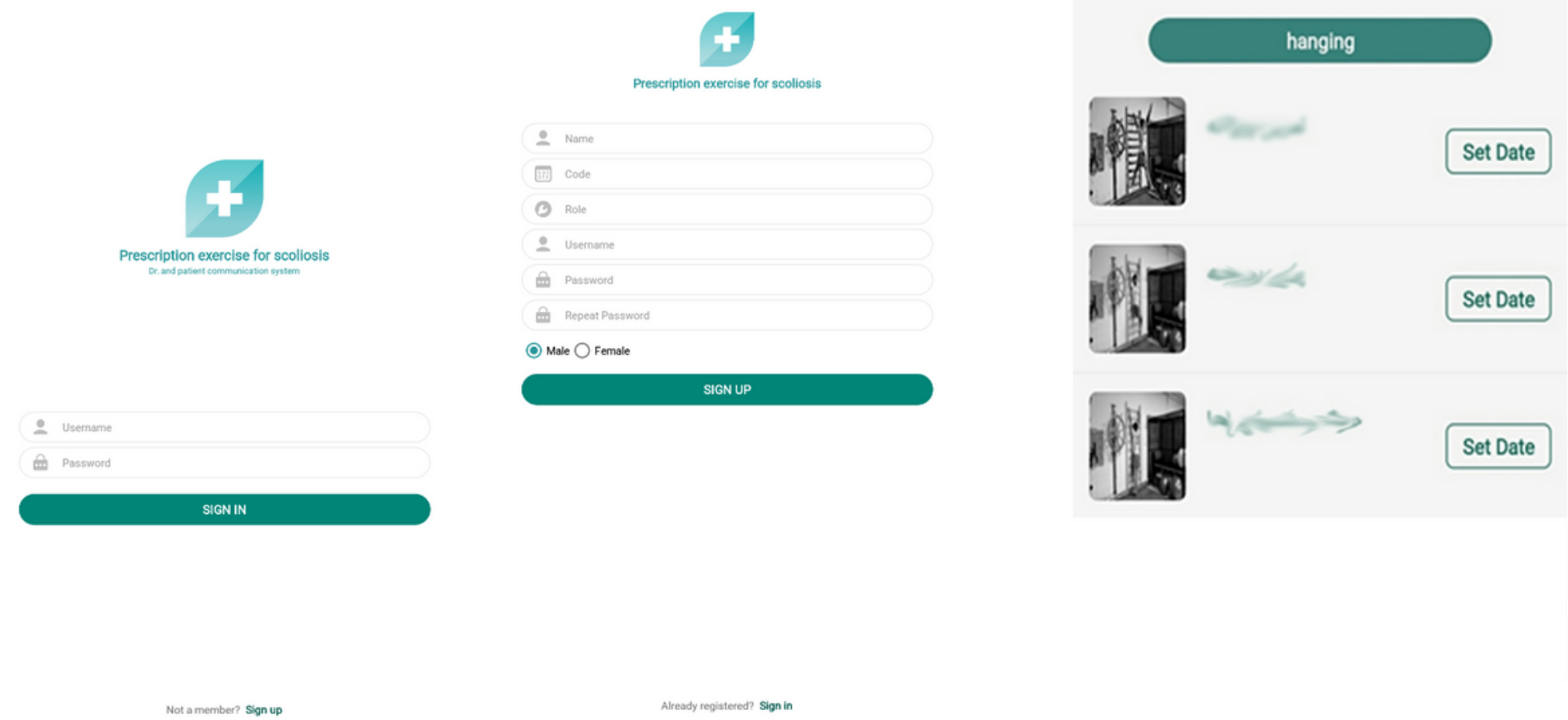

Figure 4

The screenshot of the scoliosis management app

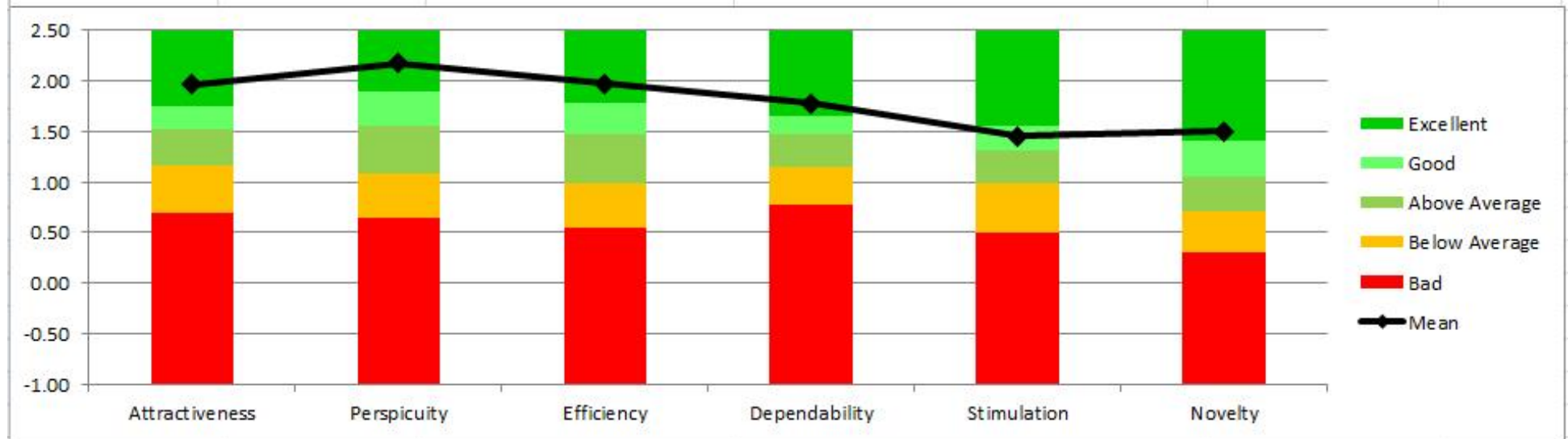

Figure 5

Users' satisfaction with the app based on UEQ 